\title{
Altered Levels of mRNAs for Calcium-Binding/Associated Proteins, Annexin A1, S100A4, and TMEM64, in Peripheral Blood Mononuclear Cells Are Associated with Osteoporosis
}

\author{
Ayed A. Dera, ${ }^{1}$ Lakshminarayan Ranganath, ${ }^{2}$ Roger Barraclough, ${ }^{3}$ Sobhan Vinjamuri, ${ }^{4}$ \\ Sandra Hamill, ${ }^{4}$ Abdullah Y. Mandourah, ${ }^{1}$ and Dong L. Barraclough $\left(\mathbb{C}^{1}\right.$ \\ ${ }^{1}$ Department of Musculoskeletal Biology, Institute of Ageing and Chronic Disease, University of Liverpool, The William Henry \\ Duncan Building, 6 West Derby Street, Liverpool L7 8TX, UK \\ ${ }^{2}$ Department of Clinical Biochemistry and Metabolic Medicine, The Royal Liverpool and Broadgreen University Hospital NHS Trust, \\ Prescot Street, Liverpool L7 8XP, UK \\ ${ }^{3}$ Department of Biochemistry, Institute of Integrative Biology, University of Liverpool, Biosciences Building, Crown Street, \\ Liverpool L69 7ZB, UK \\ ${ }^{4}$ Department of Nuclear Medicine, The Royal Liverpool and Broadgreen University Hospital NHS Trust, Prescot Street, \\ Liverpool L7 8XP, UK
}

Correspondence should be addressed to Dong L. Barraclough; dong.barraclough@liverpool.ac.uk

Received 24 July 2019; Accepted 16 September 2019; Published 11 November 2019

Academic Editor: Timo Sorsa

Copyright (C) 2019 Ayed A. Dera et al. This is an open access article distributed under the Creative Commons Attribution License, which permits unrestricted use, distribution, and reproduction in any medium, provided the original work is properly cited.

\begin{abstract}
Background. Osteoporosis is the most common metabolic bone disease in the world. Since osteoporosis is clinically symptomless until the first fracture occurs, early diagnosis is critical. Calcium, along with calcium-binding and calcium-associated proteins, plays an important role in homeostasis, maintaining healthy bone metabolism. This study is aimed at investigating the level of calcium-binding/associated proteins, annexin A1, S100A4, and TMEM64, in peripheral blood mononuclear cells associated with osteoporosis and its clinical significance. Methods. The levels of mRNAs of annexin A1, S100A4, and TMEM64 in human peripheral blood mononuclear cells were evaluated among 48 osteopenia and 23 osteoporosis patients compared to 17 nonosteoporotic controls. Total RNAs were isolated from clinical samples, and quantitation of mRNA levels was performed using real-time quantitative PCR. Results. The levels of mRNAs for calcium-binding proteins, annexin A1 and S100A4, and calcium-associated protein, TMEM64, in human peripheral blood mononuclear cells were significantly reduced in osteopenia and osteoporosis patients compared with nonosteoporotic controls (one-way ANOVA, $P<0.0001, P=0.039$, and $P=0.0195$, respectively). Annexin A1 and TMEM64 mRNAs were also significantly reduced in female osteoporosis patients over the age of 50 years compared to nonosteoporotic controls (one-way ANOVA, $P=0.004$ and $P=0.0037$, respectively). ROC analysis showed that the reduction in the level of mRNA for annexin A1, S100A4, or TMEM64 in the patients' peripheral blood mononuclear cells has a good diagnostic value for osteoporosis. Conclusions. The results show for the first time that calciumbinding/associated proteins, annexin A1 and TMEM64, could be future diagnostic biomarkers for osteoporosis.
\end{abstract}

\section{Introduction}

Osteoporosis is an age-related bone disease and has a severe impact on public health and economy worldwide, due to fragility fracture [1]. Since osteoporosis is clinically symptomless until the first fracture, there is a pressing need to identify osteoporosis at an earlier stage. Osteoporosis arises from an imbalance between bone-resorbing osteoclasts and bone-producing osteoblasts [2]. Whilst osteoblasts are derived from bone marrow cells [3], the bone-degrading osteoclasts are derived by cell fusion and differentiation of precursor monocyte blood cells [4], which can be found in the peripheral blood mononuclear cells (PBMCs) of a blood sample [5]. The PBMC fraction could be a source of markers which 
reflect changes in osteoclast activity in osteoporosis patients and possibly in those with osteopenia, the milder, early form of osteoporosis.

Calcium plays an important role in the development of osteoclasts with the involvement of calcium-binding proteins, such as annexins, S100 proteins, and proteins involved with calcium signaling, such as TMEM64. Three calcium-binding/associated proteins, annexin A1, S100A4, and TMEM64, are expressed in monocyte cells (Gene Expression Atlas: https://www.ebi.ac.uk/gxa/home). The calcium-binding, EF-hand-containing S100 protein, S100A4, has been identified in osteoclasts in developing mouse bone [6]. In mice, knockdown of S100A4 with SiRNA led to a higher bone mass and reduced numbers of osteoclasts [7], suggesting its involvement in some way with bone degradation. Furthermore, it has been suggested that S100A4 protein not only prevents bone excess but also can prevent cortical bone loss in estrogen-deprived mice [8]. Synchronised fusion of mouse osteoclasts has been reported to involve S100A4 and to depend upon a member of the calcium-dependent, phospholipid-binding annexin protein family, annexin A1 [9]. Annexin A1 is functional in the human monocytic cell line, U937 [10], reducing its vascular tethering and transmigration [11]. Furthermore, annexin A1 is an important mediator of the resolution of inflammation [12], in part by enhancing leucocyte apoptosis [13]. Inflammatory diseases have been associated with osteoporotic bone fragility [14].

The transmembrane protein, TMEM64, has been reported to interact with and modulate the activity of the protein, sarcoplasmic endoplasmic reticulum $\mathrm{Ca}^{2+}$ ATPase 2 (SERCA2), thereby enhancing RANKL-induced internal calcium oscillations that are a part of the pathway of osteoclast generation [15].

Thus, the aim of this study was to find out whether the levels of mRNAs for annexin A1, S100A4, and TMEM64 were significantly changed in the PBMCs of participants with osteopenia or with osteoporosis compared to nonosteoporotic controls.

\section{Materials and Methods}

2.1. Study Subjects. The participants in this study were men and women volunteers living in Merseyside, England, in the area covered by the Royal Liverpool and Broadgreen University Hospital NHS Trust. Participants were recruited from referrals to the Nuclear Medicine Department at the Royal Liverpool University Hospital. Diagnoses of osteopenia and osteoporosis were made in accordance with the WHO guidelines. This study conformed to the principles of the 1964 Helsinki Declaration and its later amendments or comparable ethical standards. The study was carried out under ethical approval from the England Health Research Authority National Research Ethics Service Committee, East of England-Essex (REC reference 15/EE/0051) Ethics Committees. Informed consent was obtained from all participants prior to sample collection. Details of the participants have been described elsewhere [16] (Supplementary Table S1).
2.2. Isolation of Peripheral Blood Mononuclear Cells. Peripheral blood mononuclear cells (PBMCs) were isolated from $25 \mathrm{~mL}$ blood samples and stored at $-80^{\circ} \mathrm{C}$, as described previously [16] including the final $14,000 \mathrm{~g}$ centrifugation step to yield a tight pellet of cellular material without maintaining cell viability.

2.3. Isolation and Purification of Total RNA. Total RNA from human PBMCs was extracted using a combination of TRIzol reagent and PureLink RNA mini kit (Thermo Fisher, UK), according to the manufacturer's recommendations as described previously [16]. The resulting purified RNAs were eluted in $30 \mu \mathrm{L}$ of RNase-free water and stored frozen at $-80^{\circ} \mathrm{C}$ until used. RNA concentrations and purity were measured using a Thermo Scientific NanoDrop ${ }^{\text {TM }} 2000$ spectrophotometer.

2.4. Quantitation of $m R N A$ Levels Using Real-Time Quantitative PCR (RT-qPCR). Reverse transcription reactions were carried out using the RT-First Strand kit, RTqPCR Primer Assays (Qiagen, UK), according to the manufacturer's recommendations and as described previously [16] on $500 \mathrm{ng}$ isolated RNA in a total volume of $20 \mu \mathrm{L}$ with a no reverse transcriptase, no DNA controls. Reverse transcription reactions were diluted $1: 20$, and $10 \mu \mathrm{L}$ samples were subjected to RT-qPCR for annexin A1, S100A4, TMEM64, and glyceraldehyde-3-phosphate dehydrogenase (GPDH) cDNAs, using the primers indicated in Table 1, with a SYBR Green PCR kit (Qiagen, UK) in a Roche LightCycler 96 Real-Time PCR system (Roche, UK). Each set of reactions was accompanied by the no-reverse-transcription, no-DNA control sample. The relative levels of each RNA were determined from the $\mathrm{Ct}$ value after normalization with control mRNA, GPDH using the $2^{-\Delta \Delta \mathrm{CT}}$ method [17]. Ct values $>35$ obtained from RT-qPCR were considered to be below the level of detection of the methodology.

2.5. Statistical Analyses. Statistical analysis between two groups used Student's $t$-test and between multiple groups used ANOVA with post hoc Bonferroni correction. Diagnostic values were determined using receiver operator characteristic curves. Statistical analyses were carried out using Stats Direct 3 (Altrincham, Cheshire). $P$ values of $<0.05$ were considered significant as described previously [18].

\section{Results}

3.1. The Levels of mRNAs of Calcium-Binding/Associated Proteins, Annexin A1, S100A4, and TMEM64, in the Peripheral Blood Mononuclear Cells Are Reduced in Osteoporosis Patients. The relative levels of mRNAs of calcium-binding/associated proteins, annexin A1, S100A4, and TMEM64, in isolated PBMC preparations were each significantly different between nonosteoporosis control, osteopenia, or osteoporosis groups of participants (one-way ANOVA, annexin A1 mRNA, Figure 1(a), $F(2: 85)=16.7$, $P<0.0001$; TMEM64 mRNA, Figure $1(\mathrm{~b}), F(2: 84)=4.13$, $P=0.0195 ; \mathrm{S} 100 \mathrm{~A} 4$, Figure $1(\mathrm{c}), F(2: 84)=3.4, P=0.039)$. For annexin A1 mRNA, the strongly significant decline was evident between nonosteoporotic and osteopenia groups (Figure 1(a) and Table 2; nonosteoporotic control group vs. 
TABLE 1: Qiagen primers used to amplify specific mRNAs by RT-qPCR.

\begin{tabular}{|c|c|c|c|c|}
\hline mRNA & Gene symbol & Accession number & Primer sequence & Qiagen catalogue \\
\hline Annexin A1 & ANXA1 & NM_000700.2 & $5^{\prime}$ TGTTTTAGCTCTGCTAAAAACTCCAG $3^{\prime}$ & PPH02882E \\
\hline S100 calcium-binding protein A4 & S100A4 & NM_002961 & $5^{\prime}$ CAACTTGGACAGCAACAGGGAC $3^{\prime}$ & PPH01313E \\
\hline Transmembrane protein 64 & TMEM64 & NM_001008495.3 & $5^{\prime}$ TTGGACTGCTTCCTACCCAGCTTC $3^{\prime}$ & PPH10400A \\
\hline $\begin{array}{l}\text { Glyceraldehyde-3-phosphate } \\
\text { dehydrogenase }\end{array}$ & GAPDH & NM_001256799 & $5^{\prime}$ GGCGCTGCCAAGGCTGTGGGCA 3' & PPH00150F \\
\hline
\end{tabular}

Annexin A1

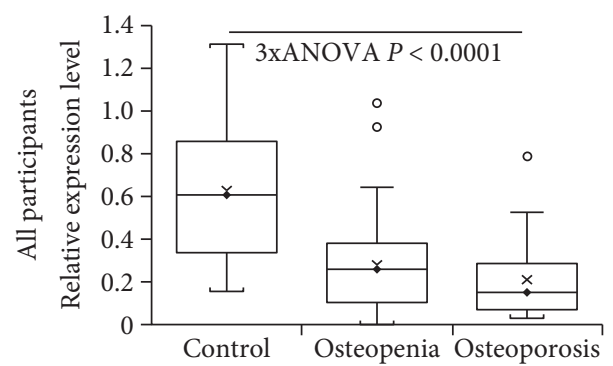

(a)

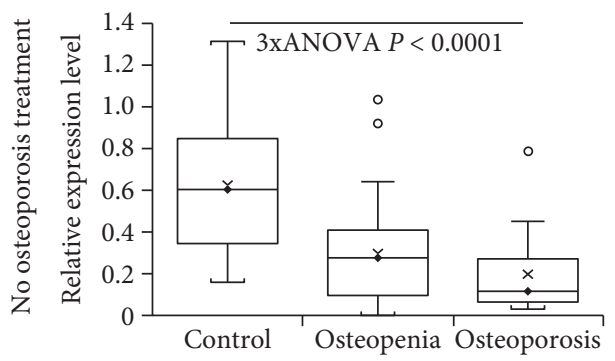

(d)

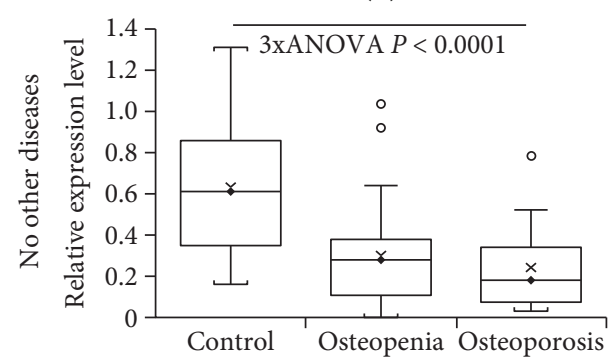

(g)

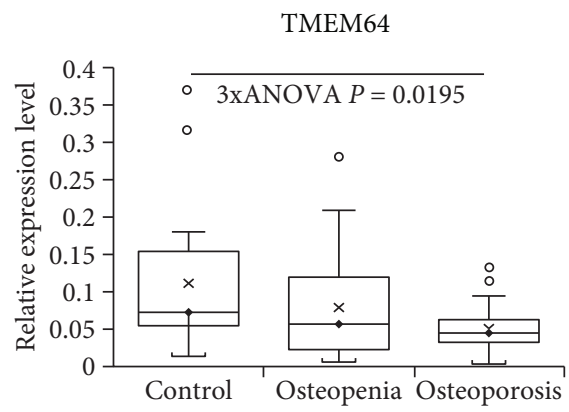

(b)

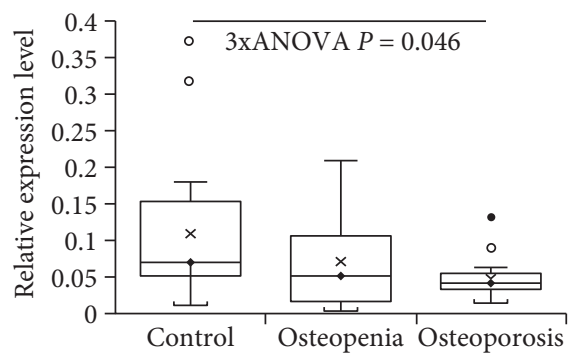

(e)

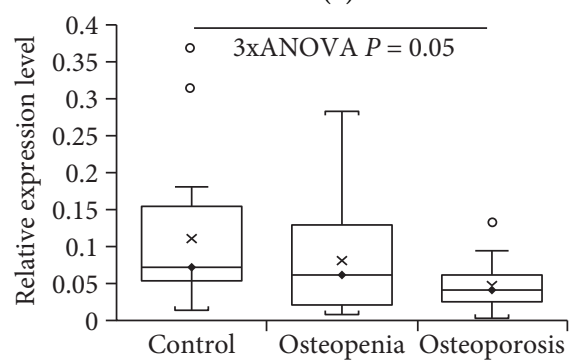

(h)

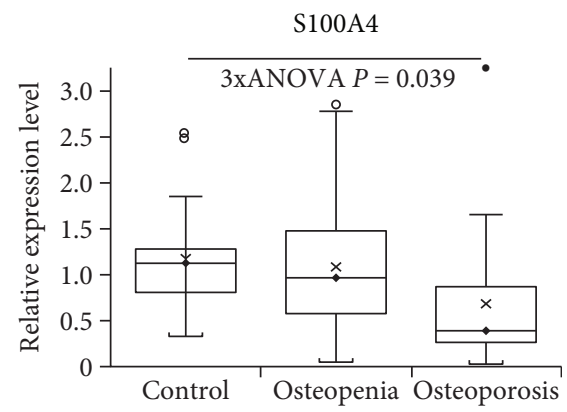

(c)

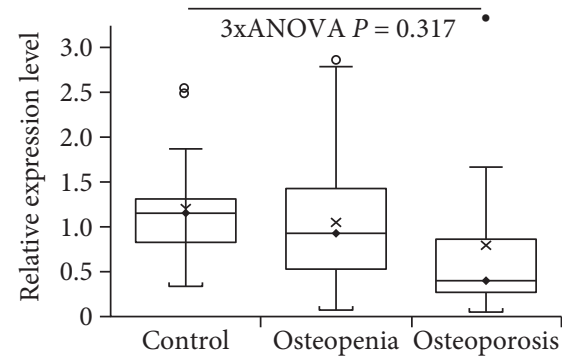

(f)

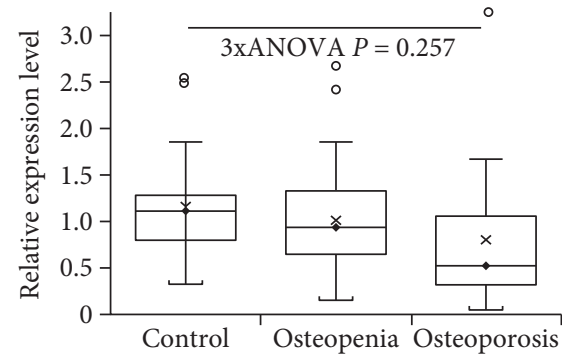

(i)

FIGURE 1: Relative levels of mRNAs in peripheral blood mononuclear cells associated with osteoporosis. Levels of mRNAs encoding annexin A1 (a, d, g), TMEM64 (b, e, h), and S100A4 (c, f, i) proteins were determined in the peripheral blood mononuclear cell preparations of nonosteoporotic control subjects and patients suffering from osteopenia or osteoporosis shown as box and whisker plots. The levels are shown for all participants (a, b, c), for participants who were not receiving treatment for osteoporosis (d, e, f), and for participants who were not suffering from other disorders $(\mathrm{g}, \mathrm{h}, \mathrm{i})$. On each box and whisker plot, the black diamond shows the median value, the cross shows the mean value, and white and black circles denote outliers of 1.5 times and 3 times the interquartile range, respectively.

osteopenia, 95\% CI 0.208 to $0.476, P<0.0001$; nonosteoporotic control group vs. osteoporosis, $95 \%$ CI 0.262 to 0.566 , $P<0.0001$; osteoporosis vs. osteopenia, 95\% CI -0.192 to $0.049, P=0.242$, post hoc Bonferroni correction). For TMEM64, there was a gradual reduction in the order: nonosteoporotic control, osteopenia, and osteoporosis groups (Figure 1(b) and Table 2; nonosteoporotic control group vs. osteopenia, $95 \%$ CI -0.004 to $0.0729, P=0.0797$; nonosteoporotic control group vs. osteoporosis, 95\% CI 0.019 to
0.106, $P=0.0052$; osteoporosis vs. osteopenia, 95\% CI -0.062 to $0.006, P=0.1$, post hoc Bonferroni correction). For S100A4 mRNA, a significant reduction was evident between osteopenia and osteoporosis groups (Figure 1(c) and Table 2; nonosteoporotic control group vs. osteopenia, $95 \%$ CI -0.302 to $0.47, P=0.666$; nonosteoporotic control group vs. osteoporosis, $95 \%$ CI 0.053 to $0.925, P=0.0286$; osteoporosis vs. osteopenia, $95 \%$ CI -0.751 to $-0.057, P=$ 0.0229 , post hoc Bonferroni correction). 
TABLE 2: Summary of statistical analysis on all participants.

\begin{tabular}{lccc}
\hline mRNA & Compared sample groups $(n=$ number of participants) & 95\% confidence interval & $P$ value (Bonferroni correction) \\
\hline \multirow{2}{*}{ Annexin A1 } & Nonosteoporotic control $(n=17)$ vs. osteopenia $(n=48)$ & 0.208 to 0.476 & $<0.0001$ \\
& Nonosteoporotic control $(n=17)$ vs. osteoporosis $(n=23)$ & 0.262 to 0.566 & $<0.0001$ \\
& Osteoporosis $(n=23)$ vs. osteopenia $(n=48)$ & -0.192 to 0.049 & 0.242 \\
\hline \multirow{2}{*}{ TMEM64 } & Nonosteoporotic control $(n=16)$ vs. osteopenia $(n=48)$ & -0.004 to 0.0729 & 0.0797 \\
& Nonosteoporotic control $(n=16)$ vs. osteoporosis $(n=23)$ & 0.019 to 0.106 & 0.0052 \\
\hline \multirow{2}{*}{ S100A4 } & Osteoporosis $(n=23)$ vs. osteopenia $(n=48)$ & -0.062 to 0.006 & 0.1 \\
& Nonosteoporotic control $(n=17)$ vs. osteopenia $(n=47)$ & -0.302 to 0.47 & 0.666 \\
& Nonosteoporotic control $(n=17)$ vs. osteoporosis $(n=23)$ & 0.053 to 0.925 & 0.0286 \\
\hline
\end{tabular}

3.2. Analysis of Levels of mRNAs for CalciumBinding/Associated Proteins Associated with Treatment of Osteoporosis and Other Disorders. For annexin A1 or TMEM64, the significant associations of reduced levels of mRNA with osteopenia and osteoporosis were not affected when participants receiving treatment for osteoporosis were excluded from the analysis (annexin A1 mRNA, one-way ANOVA, $F(2,68)=12.7, \quad P<0.0001 ;$ Figure $1(\mathrm{~d})$ and Table 3; nonosteoporotic control group vs. osteopenia, $95 \%$ CI 0.18 to $0.478, P<0.0001$; nonosteoporotic control group vs. osteoporosis, $95 \%$ CI 0.232 to $0.603, P<0.0001$; osteoporosis vs. osteopenia, $95 \% \mathrm{CI}-0.248$ to $0.071, P=0.272$; TMEM64 mRNA, one-way ANOVA, $\quad F(2,67)=3.2, \quad P=0.046$; Figure 1(e) and Table 3; nonosteoporotic control group vs. osteopenia, $95 \%$ CI -0.00096 to $0.0784, P=0.056$; nonosteoporotic control group vs. osteoporosis, 95\% CI 0.011 to $0.109, P=0.0168$; osteoporosis vs. osteopenia, 95\% CI -0.063 to $0.02, P=0.305$; Bonferroni post hoc test). When participants with nonosteoporotic medical disorders were excluded, for annexin A1 or TMEM64, the significant associations of reduced levels of mRNA with osteopenia and osteoporosis were not affected (annexin A1 mRNA, one-way ANOVA, $F(2,66)=11.8, \quad P<0.0001$; Figure $1(\mathrm{~g})$ and Table 4; nonosteoporotic control group vs. osteopenia, 95\% CI 0.176 to $0.477, P<0.0001$; nonosteoporotic control group vs. osteoporosis, 95\% CI 0.202 to $0.557, P<0.0001$; osteoporosis vs. osteopenia, $95 \%$ CI -0.206 to $0.1, P=0.491$; TMEM mRNA, one-way ANOVA, $F(2,65)=3.1, P=0.05$; Figure $1(\mathrm{~h})$ and Table 4; nonosteoporotic control group vs. osteopenia, $95 \%$ CI -0.013 to $0.075, P=0.166$; nonosteoporotic control group vs. osteoporosis, $95 \%$ CI 0.013 to 0.117 , $P=0.016$; osteoporosis vs. osteopenia, 95\% CI -0.078 to $0.01, P=0.132$, Bonferroni post hoc test).

However, for S100A4, the significant association between the reduction in S100A4 mRNA and osteoporosis was lost when participants either receiving treatment for osteoporosis or participants with nonosteoporotic medical disorders were excluded from the analysis (osteoporosis treatment removed, one-way ANOVA, $F(2,67)=1.17, P=0.317$; Figure $1(\mathrm{f})$ and Table 3; other diseases removed one-way ANOVA, $F(2,66)$ $=1.39, P=0.257$; Figure 1(i) and Table 4), showing that the relationship between S100A4 mRNA level and osteoporosis was potentially affected by treatment for osteoporosis and by the presence of other disorders, whereas the relation- ship between annexin A1 or TMEM64 mRNA and osteoporosis was not.

3.3. The Levels of mRNAs of Calcium-Binding/Associated Proteins Are Reduced in the Peripheral Blood Mononuclear Cells of Female Osteoporosis Patients Aged over 50 Years. To find out whether the same results were obtained in female osteoporosis patients aged over 50 years specifically, the analyses were confined to the 63 female subjects over 50 years of age. All three mRNAs showed the same trend of a decrease in the mRNA level in the order: nonosteoporotic control, osteopenia, and osteoporosis observed when all patients were included. Annexin A1 and TMEM64 mRNAs showed a significant reduction between nonosteoporotic, osteopenia, and osteoporosis participants (annexin A1 mRNA, one-way ANOVA, $F(2,60)=5.95, P=0.004$; Figure 2(a) and Table 5; nonosteoporotic controls vs. osteopenia, $95 \%$ CI 0.114 to $0.493, P=0.0021$; nonosteoporotic control group vs. osteoporosis, 95\% CI 0.125 to 0.539 , $P=0.0022$; osteoporosis vs. osteopenia, 95\% CI -0.175 to $0.118, P=0.699$, Bonferroni post hoc test; TMEM64 mRNA, one-way ANOVA, $F(2,60)=6.14, P=0.0037$; Figure 2(b) and Table 5; nonosteoporotic control group vs. osteopenia, 95\% CI 0.008 to $0.108, P=0.0245$; nonosteoporotic control group vs. osteoporosis, 95\% CI 0.041 to $0.15, P=0.0009$; osteoporosis vs. osteopenia, $95 \%$ CI -0.077 to 0.0009 , $P=0.056$, Bonferroni post hoc test). However, the changes in S100A4 mRNA between the nonosteoporotic control group, osteopenia, or osteoporosis patients were not significant in the female participants aged over 50 years (one-way ANOVA, $F(2,59)=2.08, P=0.134$; Figure 2(c) and Table 5).

3.4. The Levels of $m$ RNAs for Annexin A1, TMEM64, or S100A4 in Peripheral Blood Mononuclear Cells from Participants with or without Chronic Inflammatory Disorders. Osteoporosis has been associated with chronic inflammatory disorders. In order to find out whether the reduction in the levels of mRNAs for annexin A1, TMEM64, or S100A4 in peripheral blood mononuclear cells was associated with the presence of chronic diseases, the participants with osteopenia or osteoporosis were divided into those without and those with reported chronic diseases or undergoing treatment with steroids. The levels of annexin A1, TMEM64, or S100A4 mRNAs in PBMCs were not significantly different between 
TABLE 3: Summary of statistical analysis on participants with no osteoporosis treatment.

\begin{tabular}{lccc}
\hline mRNA & Compared sample groups $(n=$ number of participants) & 95\% confidence interval & $P$ value (Bonferroni correction) \\
\hline \multirow{2}{*}{ Annexin A1 } & Nonosteoporotic control $(n=17)$ vs. osteopenia $(n=40)$ & 0.18 to 0.478 & $<0.0001$ \\
& Nonosteoporotic control $(n=17)$ vs. osteoporosis $(n=14)$ & 0.232 to 0.603 & $<0.0001$ \\
& Osteoporosis $(n=14)$ vs. osteopenia $(n=40)$ & -0.248 to 0.071 & 0.272 \\
\hline \multirow{2}{*}{ TMEM64 } & Nonosteoporotic control $(n=16)$ vs. osteopenia $(n=40)$ & -0.00096 to 0.0784 & 0.056 \\
& Nonosteoporotic control $(n=16)$ vs. osteoporosis $(n=14)$ & 0.011 to 0.109 & 0.0168 \\
\hline \multirow{2}{*}{ S100A4 } & Osteoporosis $(n=14)$ vs. osteopenia $(n=40)$ & -0.063 to 0.02 & 0.305 \\
& Nonosteoporotic control $(n=17)$ vs. osteopenia $(n=39)$ & -0.273 to 0.556 & 0.498 \\
& Nonosteoporotic control $(n=17)$ vs. osteoporosis $(n=14)$ & -0.124 to 0.906 & 0.134 \\
\hline
\end{tabular}

TABLE 4: Summary of statistical analysis on participants without nonosteoporotic medical disorders.

\begin{tabular}{lccc}
\hline mRNA & Compared sample groups $(n=$ number of participants) & $95 \%$ confidence interval & $P$ value (Bonferroni correction) \\
\hline \multirow{2}{*}{ Annexin A1 } & Nonosteoporotic control $(n=17)$ vs. osteopenia $(n=36)$ & 0.176 to 0.477 & $<0.0001$ \\
& Nonosteoporotic control $(n=17)$ vs. osteoporosis $(n=16)$ & 0.202 to 0.557 & $<0.0001$ \\
& Osteoporosis $(n=16)$ vs osteopenia $(n=36)$ & -0.206 to 0.1 & 0.491 \\
\hline \hline \multirow{2}{*}{ TMEM64 } & Nonosteoporotic control $(n=16)$ vs. osteopenia $(n=36)$ & -0.013 to 0.075 & 0.166 \\
& Nonosteoporotic control $(n=16)$ vs. osteoporosis $(n=16)$ & 0.013 to 0.117 & 0.016 \\
\hline \multirow{2}{*}{ S100A4 } & Osteoporosis $(n=16)$ vs osteopenia $(n=36)$ & -0.078 to 0.01 & 0.132 \\
& Nonosteoporotic control $(n=17)$ vs. osteopenia $(n=36)$ & -0.224 to 0.54 & 0.413 \\
& Nonosteoporotic control $(n=17)$ vs. osteoporosis $(n=16)$ & -0.077 to 0.828 & 0.102 \\
\hline
\end{tabular}

Annexin A1

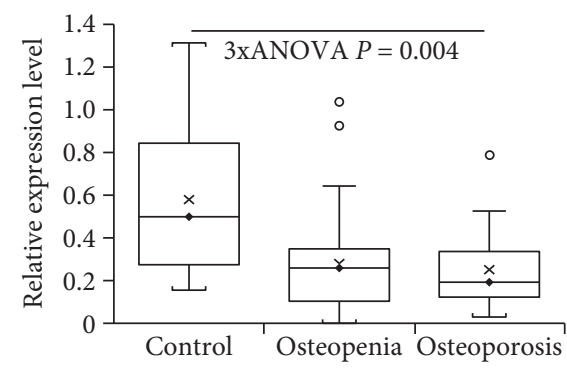

(a)

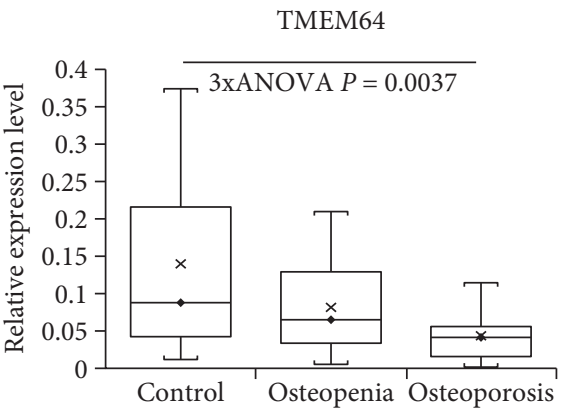

(b)

S100A4

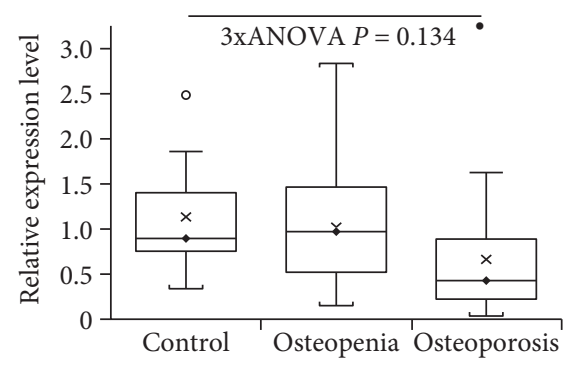

(c)

FIGURE 2: Relative levels of mRNAs in peripheral blood mononuclear cells associated with osteoporosis in females over the age of 50. Levels of mRNAs encoding annexin A1 (a), TMEM64 (b), and S100A4 (c) proteins were determined in peripheral blood mononuclear cell preparations of nonosteoporotic control subjects and patients suffering from osteopenia or osteoporosis. On each box and whisker plot, the black diamond shows the median value, the cross shows the mean value, and white and black circles denote outliers of 1.5 times and 3 times the interquartile range, respectively. 
TABLE 5: Summary of statistical analysis on female participants over the age of 50.

\begin{tabular}{lccc}
\hline mRNA & Compared sample groups $(n=$ number of participants) & 95\% confidence interval & $P$ value (Bonferroni correction) \\
\hline \multirow{2}{*}{ Annexin A1 } & Nonosteoporotic control $(n=9)$ vs. osteopenia $(n=36)$ & 0.114 to 0.493 & 0.0021 \\
& Nonosteoporotic control $(n=9)$ vs. osteoporosis $(n=18)$ & 0.125 to 0.539 & 0.0022 \\
& Osteoporosis $(n=18)$ vs. osteopenia $(n=36)$ & -0.175 to 0.118 & 0.699 \\
\hline \multirow{2}{*}{ TMEM64 } & Nonosteoporotic control $(n=9)$ vs. osteopenia $(n=36)$ & 0.008 to 0.108 & 0.0245 \\
& Nonosteoporotic control $(n=9)$ vs. osteoporosis $(n=18)$ & 0.041 to 0.15 & 0.0009 \\
\hline \multirow{2}{*}{ S100A4 } & Osteoporosis $(n=18)$ vs. osteopenia $(n=36)$ & -0.077 to 0.0009 & 0.056 \\
& Nonosteoporotic control $(n=9)$ vs. osteopenia $(n=35)$ & -0.406 to 0.601 & 0.7 \\
& Nonosteoporotic control $(n=9)$ vs. osteoporosis $(n=18)$ & -0.095 to 1.01 & 0.103 \\
\hline
\end{tabular}

those with and those without known chronic inflammatory disorders (Supplementary Figure S1).

\subsection{Diagnostic Value of Calcium-Binding/Associated Protein} $m R N A s$ for Osteoporosis. The levels of mRNAs for annexin A1, S100A4, and TMEM64 were reduced in PBMCs from osteoporosis patients compared to nonosteoporotic controls; thus, the presence of these mRNAs marked the nonosteoporotic condition. ROC curve analysis for nonosteoporotic controls and osteoporosis showed that, when all participants were included, the areas under the ROC curves for annexin A1, TMEM64, and S100A4 were 0.893, (Figure 3(a), 95\% CI 0.796 to 0.99 ; sensitivity 0.882 , specificity 0.783 ), 0.742 (Figure 3(b), 95\% CI 0.577 to 0.907 ; sensitivity 0.813 , specificity 0.696 ), and 0.783 (Figure 3 (c), 95\% CI 0.637 to 0.929 ; sensitivity 0.882 , specificity 0.652 ), respectively. The reduction of neither TMEM64 nor S100A4 discriminated well nonosteoporotic controls from osteopenia (area under the ROC curves, 0.6 and 0.55 , respectively, not shown) or osteopenia and osteoporosis combined (area under the ROC curves, 0.646 and 0.627 , respectively, not shown). However, for annexin A1, when nonosteoporotic controls were compared with osteopenia, the area under the ROC curve was 0.805 (Figure $4(\mathrm{a}), 95 \%$ CI 0.682 to 0.928 ; sensitivity 0.588 , specificity 0.917 ) and when compared with osteopenia and osteoporosis combined, the area under the ROC curve was 0.833 (Figure 4(b), 95\% CI 0.729 to 0.938 ; sensitivity 0.588 , specificity 0.93 ).

When the analysis was confined to females over the age of 50 years, similar results were obtained, with areas under the ROC curves for annexin A1, TMEM64, and S100A4 mRNAs being 0.809 (Figure 3(d), 95\% CI 0.635 to 0.983 ; sensitivity 0.778 , specificity 0.722 ), 0.765 (Figure 3(e), 95\% CI 0.541 to 0.99 ; sensitivity 0.778 , specificity 0.722 ), and 0.765 (Figure 3(f), 95\% CI 0.583 to 0.948 ; sensitivity 1.0 , specificity 0.444), respectively. Neither TMEM64 nor S100A4 mRNAs discriminated well nonosteoporotic controls from osteopenia (area under the ROC curves, 0.614 and 0.543, respectively, not shown) or osteopenia and osteoporosis combined (area under the ROC curves, 0.667 and 0.618 , respectively, not shown). However, for annexin A1 mRNA, when nonosteoporotic controls were compared with osteopenia, the area under the curve was 0.747 (Figure 4(c), 95\% CI 0.563 to 0.931 ; sensitivity 0.556 , specificity 0.861 ) and when com- pared with osteopenia and osteoporosis combined, the area under the curve was 0.767 (Figure 4(d), 95\% CI 0.598 to 0.937 ; sensitivity 0.556 , specificity 0.87 ).

\section{Discussion}

Three mRNAs, encoding calcium-binding/associated proteins, ANXA1, S100A4, and TMEM64, were found to be at a lower level in the PBMC preparations from osteoporosis patients than nonosteoporotic controls. The reductions of S100A4 mRNA levels were found to be due to participants with other disorders, those receiving existing treatments for osteoporosis, males, and under 50-year olds. In contrast, the observed reductions in annexin A1 or TMEM64 mRNAs were not affected by any of these factors.

Annexin A1 protein and, to a lesser extent, TMEM64 protein are found in the CD14+/CD16-negative monocytes [19] (Gene Expression Atlas: https://www.ebi.ac.uk/gxa/ home). These cells have been shown to be the likely source of osteoclasts by differentiation in vitro of cells from normal human subjects [20]. For TMEM64 mRNA in PBMCs, the observed reduction in the level with osteoporosis in the present experiments seems unexpected in view of previous results with TMEM64 knockout mice, in which reduced levels of TMEM64 mRNA resulted in a phenotype of increased bone mass [21]. Apart from possible species differences between mouse and human, such as observed for annexin A1 [9], the difference between the present results and the TMEM64 mouse knockout results could arise from the cell preparations being examined in the two studies. In the knockout mice, there was a reduction in bone-associated, fully differentiated osteoclasts [21], whereas in the present study, the TMEM64 mRNA levels were examined in the blood PBMC fraction, which contains monocytes as yet undifferentiated into osteoclasts [4].

Annexin A1 has been reported to be present in the human U937 cultured monocyte cell line, where it has been reported to reduce adhesion of U937 cells to bone marrow endothelial cells by directly interacting with $\alpha 4$ integrin and competing with binding of the endothelial $\alpha 4$ integrin receptor, VCAM-1 [11]. A reduction in annexin A1 mRNA, and thus possibly a reduction in annexin A1 protein, in monocytes could therefore result in increased retention of 


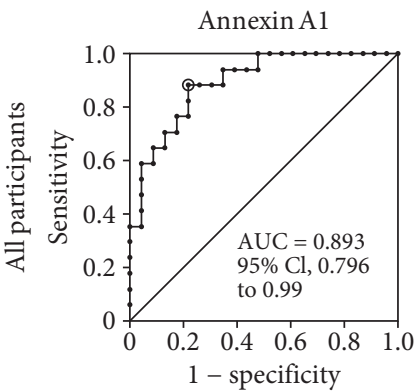

(a)

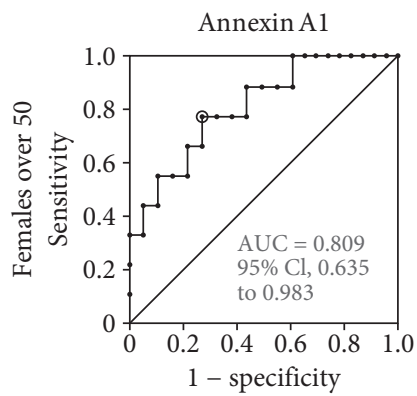

(d)

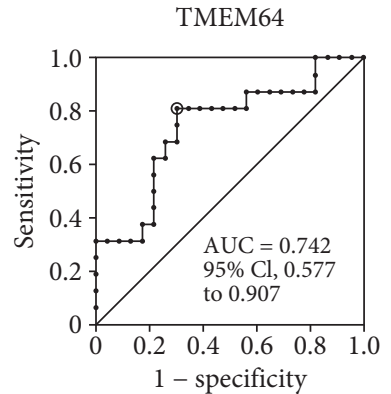

(b)

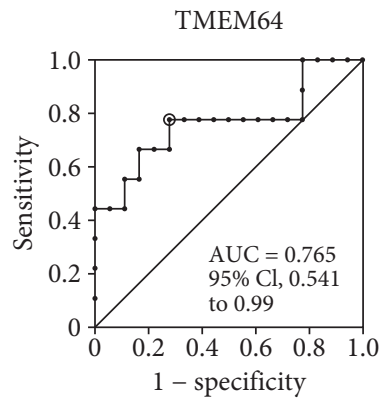

(e)

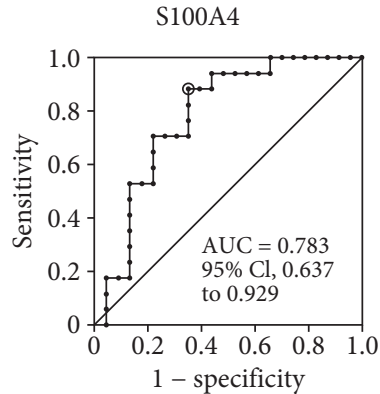

(c)

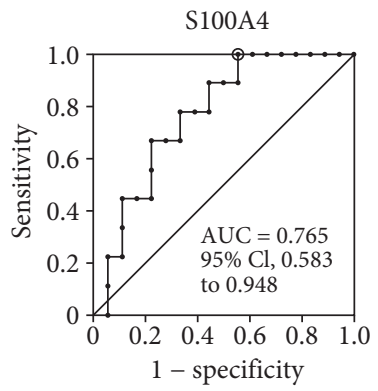

(f)

Figure 3: Diagnostic values of annexin A1, TMEM64, and S100A4 mRNAs for nonosteoporotic controls vs osteoporosis. ROC curves between nonosteoporotic controls and osteoporosis sufferers are shown for the mRNAs of annexin A1 (a, d), TMEM64 (b, e), and S100A4 $(c, f)$ for all participants $(a, b, c)$ and female participants over the age of 50 (d, e, f). The circled point indicates the optimum cut-off.

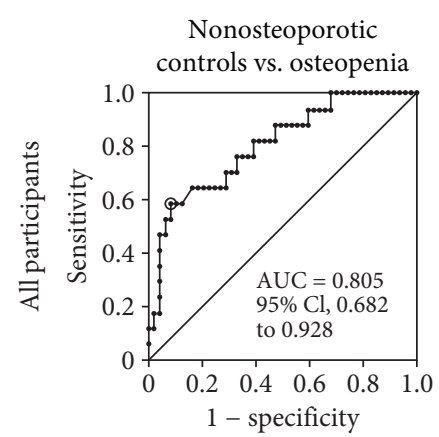

(a)

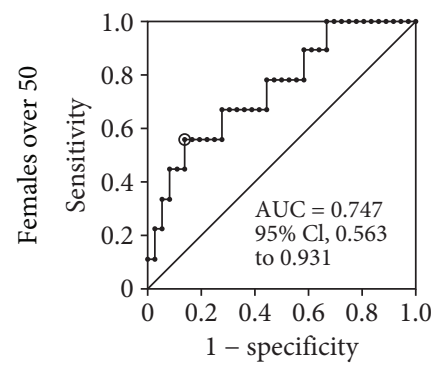

(c)

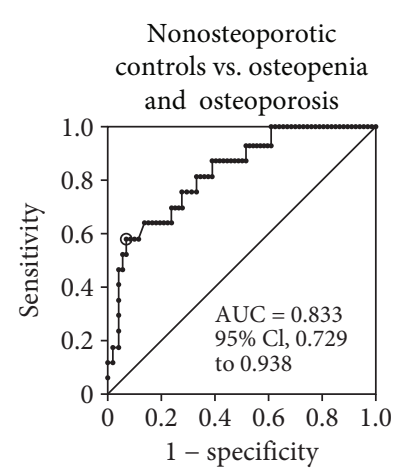

(b)

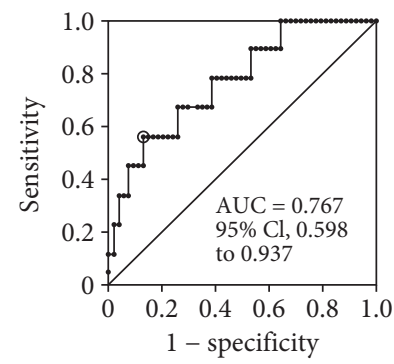

(d)
FIgURE 4: Diagnostic values of annexin A1 for nonosteoporotic controls vs osteopenia and osteoporosis. ROC curves are shown for the mRNA for annexin A1 for all participants (a, b) and female participants over the age of 50 (c, d), comparing nonosteoporotic controls with osteopenia ( $a, c)$ and nonosteoporotic controls with osteopenia and osteoporosis (b, d). The circled point indicates the optimum cut-off. monocytes by endothelial cells in the bone marrow and reduced numbers in peripheral blood mononuclear cells.

However, the PBMC fractions consist of only 20-40\% monocytes and a low percentage of polymorphonuclear cells (1-4\%), but a majority (60-80\%) of cells in PBMC preparations are lymphocytes [22]. TMEM64 mRNA is present at a low level in both $\mathrm{T}$ and $\mathrm{B}$ lymphocytes and is more abundant in natural killer cells (Gene Expression Atlas: https://www.ebi.ac.uk/gxa/ home). Annexin A1 (lipocortin 1) has been reported to be present at a consistent level in many $\mathrm{T}$ lymphocyte subtypes and at a higher level in natural killer cells [19], but not present in B lymphocytes (Gene Expression Atlas: https://www.ebi.ac.uk/gxa/home). Thus, it is possible that the observed reductions in annexin A1 and TMEM64 mRNAs arise in the lymphocyte populations in the PBMC preparations. It is not known whether there are specific differences in the cell types populating PBMCs of nonosteoporotic individuals and osteopenia and osteoporosis patients. However, T lymphocytes $[23,24]$ and natural killer cells [25] have been reported to contribute to osteoporosis by their activation and production of osteoclastogenic cytokines, but the observation that various $\mathrm{T}$ cell subtypes can exhibit either osteoclastogenic, e.g., Thy 17, or antiosteoclastogenic activity, e.g., Thy1 [26], suggests that there is a balance between pro- and antiosteoclastic activity in $\mathrm{T}$ cell subtypes. Whilst, little, if anything, is known about any possible role of TMEM64 in these processes in T lymphocytes, the presence of annexin A1, on the other hand, has been widely associated with the resolution of inflammatory disorders $[12,13,27]$. Thus, a change in the balance of $\mathrm{T}$ cell osteoclastogenic activity, arising from the reduced annexin 
A1 mRNA levels in PBMCs from osteopenia/osteoporosis patients, could contribute to their osteopenia/osteoporosis. However, this might not be consistent with the observation on the present group of participants that the levels of annexin mRNA in the PBMCs of participants with chronic inflammatory disorders were not significantly different from those without chronic inflammatory disorders, either when all participants or when only the female-over-50 group were considered.

ROC curve analysis for the changes in these mRNAs showed moderate accuracy for the areas under the curves [28]. Annexin A1 exhibited the best trade-off of sensitivity against specificity of the three possible markers for all participants when nonosteoporotic controls were compared with osteoporosis (area under the curve, 0.893), with osteopenia (area under the curve, 0.805 ), or with osteopenia and osteoporosis combined (area under the curve, 0.833 ) or when only females over the age of 50 were included (areas under the curves, $0.809,0.747$, and 0.767 , respectively).

In conclusion, this study demonstrates for the first time that the levels of mRNAs of annexin A1 and TMEM64 in peripheral blood mononuclear cells are reduced in association with osteoporosis, at least in this group of patients with loss of annexin A1 mRNA in peripheral blood mononuclear cells being a good marker for osteopenia/osteoporosis.

\section{Data Availability}

The data used to support the findings of this study are available from the corresponding author upon request.

\section{Ethical Approval}

All procedures performed in studies involving human participants were in accordance with the ethical standards of the England Health Research Authority National Research Ethics Service Committee, East of England-Essex (REC reference 15/EE/0051) Ethics Committee and with the 1964 Helsinki declaration and its later amendments or comparable ethical standards.

\section{Disclosure}

The current address of Abdullah Y. Mandourah is Al Hada Armed Forces Hospital, Taif, Saudi Arabia. The current address of Ayed Dera is Department of Clinical Laboratory Sciences, College of Applied Medical Sciences, King Khalid University, Abha, Saudi Arabia.

\section{Conflicts of Interest}

The authors declare that they have no conflicts of interest.

\section{Authors' Contributions}

D.L.B. conceived the study and designed the experiments. A.A.D. and A.Y.M. performed the experiments; A.A.D. analysed the data. L.R., R.B., S.V., and S.H. provided intellectual guidance in the study and interpretation of data. D.L.B. and R.B. wrote and prepared the manuscript with contribution from all coauthors. All the authors have accepted responsibility for the entire content of this submitted manuscript and approved submission.

\section{Acknowledgments}

We thank the healthy volunteers and patients who participated in this study. We thank the members of the Unit of Clinical Chemistry and Department of Nuclear Medicine at the Royal Liverpool and Broadgreen University Hospital NHS Trust and Institute of Ageing and Chronic Disease at the University of Liverpool for all their support. A.A.D. and A.Y.M. were supported by Ph.D. scholarships from the Saudi Arabian Embassy.

\section{Supplementary Materials}

Supplementary Figure S1: relative levels of mRNAs for annexin A1, TMEM64, and S100A4 were determined in the peripheral blood mononuclear cells of osteopenia and osteoporosis participants. Box and whisker plots show the levels of mRNAs in participants with or without chronic inflammatory disorders/treatment with steroids. Supplementary Table S1: summary of characteristics of clinical samples. (Supplementary Materials)

\section{References}

[1] N. Harvey, E. Dennison, and C. Cooper, "Osteoporosis: impact on health and economics," Nature Reviews Rheumatology, vol. 6 , no. 2, pp. 99-105, 2010.

[2] X. Feng and J. M. McDonald, "Disorders of bone remodeling," Annual Review of Pathology, vol. 6, pp. 121-145, 2011.

[3] J. Y. Wu, D. T. Scadden, and H. M. Kronenberg, "Role of the osteoblast lineage in the bone marrow hematopoietic niches," Journal of Bone and Mineral Research, vol. 24, no. 5, pp. 759-764, 2009.

[4] J. M. W. Quinn, S. Neale, Y. Fujikawa, and J. O. D. McGee, "Human osteoclast formation from blood monocytes, peritoneal macrophages, and bone marrow cells," Calcified Tissue International, vol. 62, no. 6, pp. 527-531, 1998.

[5] G. C. Nicholson, M. Malakellis, F. M. Collier et al., "Induction of osteoclasts from CD14-positive human peripheral blood mononuclear cells by receptor activator of nuclear factor $\kappa \mathrm{B}$ ligand (RANKL)," Clinical Science, vol. 99, no. 2, pp. 133140,2000

[6] J. Klingelhofer, N. S. Ambartsumian, and E. M. Lukanidin, "Expression of the metastasis-associated $m t s 1$ gene during mouse development," Developmental Dynamics, vol. 210, no. 2, pp. 87-95, 1997.

[7] M. C. Erlandsson, M. D. Svensson, I.-M. Jonsson et al., "Expression of metastasin S100A4 is essential for bone resorption and regulates osteoclast function," Biochimica et Biophysica Acta (BBA) - Molecular Cell Research, vol. 1833, no. 12, pp. 2653-2663, 2013.

[8] M. C. Erlandsson, L. Bian, I.-M. Jonsson, K. M. Andersson, and M. I. Bokarewa, "Metastasin S100A4 is a mediator of sex hormone-dependent formation of the cortical bone," Molecular Endocrinology, vol. 27, no. 8, pp. 1311-1321, 2013. 
[9] S. K. Verma, E. Leikina, K. Melikov et al., "Cell-surface phosphatidylserine regulates osteoclast precursor fusion," Journal of Biological Chemistry, vol. 293, no. 1, pp. 254-270, 2018.

[10] C. Sundstrom and K. Nilsson, "Establishment and characterization of a human histiocytic lymphoma cell line (U-937)," International Journal of Cancer, vol. 17, pp. 565-577, 1976.

[11] E. Solito, I. A. Romero, S. Marullo, F. Russo-Marie, and B. B. Weksler, "Annexin 1 binds to U937 monocytic cells and inhibits their adhesion to microvascular endothelium: involvement of the $\alpha_{4} \beta_{1}$ integrin," The Journal of Immunology, vol. 165, no. 3, pp. 1573-1581, 2000.

[12] T. Gobbetti and S. N. Cooray, "Annexin A1 and resolution of inflammation: tissue repairing properties and signalling signature," Biological Chemistry, vol. 397, no. 10, pp. 981-993, 2016.

[13] J. P. Vago, C. R. C. Nogueira, L. P. Tavares et al., “Annexin A1 modulates natural and glucocorticoid-induced resolution of inflammation by enhancing neutrophil apoptosis," Journal of Leukocyte Biology, vol. 92, no. 2, pp. 249-258, 2012.

[14] K. Briot, P. Geusens, I. E. Bultink, W. F. Lems, and C. Roux, "Inflammatory diseases and bone fragility," Osteoporosis International, vol. 28, no. 12, pp. 3301-3314, 2017.

[15] Y. M. Yang, M. S. Kim, A. Son et al., "Alteration of RANKLinduced osteoclastogenesis in primary cultured osteoclasts from SERCA $2^{+/-}$mice," Journal of Bone and Mineral Research, vol. 24, no. 10, pp. 1763-1769, 2009.

[16] A. A. Dera, L. Ranganath, R. Barraclough, S. Vinjamuri, S. Hamill, and D. L. Barraclough, "Cathepsin Z as a novel potential biomarker for osteoporosis," Scientific Reports, vol. 9, no. 1, p. 9752, 2019.

[17] K. J. Livak and T. D. Schmittgen, "Analysis of relative gene expression data using real-time quantitative PCR and the $2^{-\Delta \Delta \mathrm{C}}$ method," Methods, vol. 25, no. 4, pp. 402-408, 2001.

[18] A. Y. Mandourah, L. Ranganath, R. Barraclough et al., "Circulating microRNAs as potential diagnostic biomarkers for osteoporosis," Scientific Reports, vol. 8, no. 1, p. 8421, 2018.

[19] E. F. Morand, P. Hutchinson, A. Hargreaves, N. J. Goulding, N. W. Boyce, and S. R. Holdsworth, "Detection of intracellular lipocortin 1 in human leukocyte subsets," Clinical Immunology and Immunopathology, vol. 76, no. 2, pp. 195-202, 1995.

[20] Y. Komano, T. Nanki, K. Hayashida, K. Taniguchi, and N. Miyasaka, "Identification of a human peripheral blood monocyte subset that differentiates into osteoclasts," Arthritis Research \& Therapy, vol. 8, no. 5, article R152, 2006.

[21] H. Kim, T. Kim, B.-C. Jeong et al., "Tmem64 modulates calcium signaling during RANKL-mediated osteoclast differentiation," Cell Metabolism, vol. 17, no. 2, pp. 249-260, 2013.

[22] A. J. Ulmer, W. Scholz, M. Ernst, E. Brandt, and H.-D. Flad, "Isolation and subfractionation of human peripheral blood mononuclear cells (PBMC) by density gradient centrifugation on Percoll," Immunobiology, vol. 166, no. 3, pp. 238-250, 1984.

[23] S. L. Teitelbaum, "Postmenopausal osteoporosis, T cells, and immune dysfunction," Proceedings of the National Academy of Sciences of the United States of America, vol. 101, no. 48, pp. 16711-16712, 2004.

[24] P. D'Amelio, A. Grimaldi, S. Di Bella et al., "Estrogen deficiency increases osteoclastogenesis up-regulating T cells activity: a key mechanism in osteoporosis," Bone, vol. 43 , no. 1 , pp. 92-100, 2008.

[25] K. Tilkeridis, G. Kiziridis, A. Ververidis et al., "Immunoporosis: a new role for invariant natural killer T (NKT) cells through overexpression of nuclear factor $-\kappa \mathrm{B}$ ligand
(RANKL)," Medical Science Monitor, vol. 25, pp. 2151-2158, 2019.

[26] R. K. Srivastava, H. Y. Dar, and P. K. Mishra, "Immunoporosis: immunology of osteoporosis-role of T cells," Frontiers in Immunology, vol. 9, p. 657, 2018.

[27] A. Sena, I. Grishina, A. Thai et al., "Dysregulation of antiinflammatory annexin A1 expression in progressive Crohns disease," PLoS One, vol. 8, no. 10, article e76969, 2013.

[28] A. K. Akobeng, "Understanding diagnostic tests 3: receiver operating characteristic curves," Acta Paediatrica, vol. 96, no. 5, pp. 644-647, 2007. 


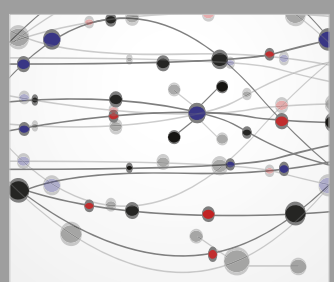

The Scientific World Journal
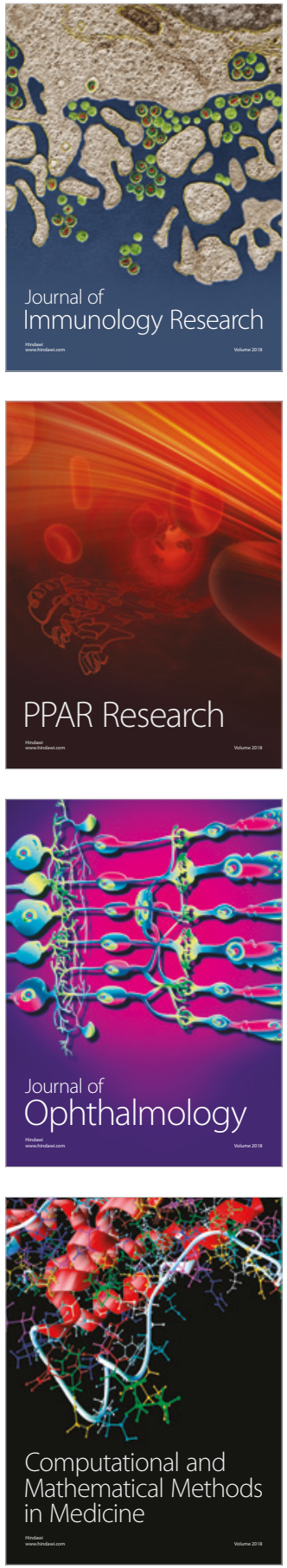

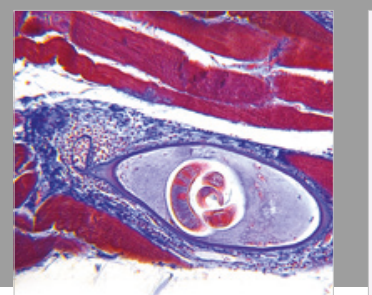

Gastroenterology Research and Practice

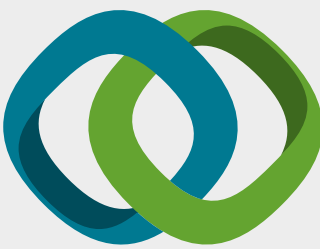

\section{Hindawi}

Submit your manuscripts at

www.hindawi.com
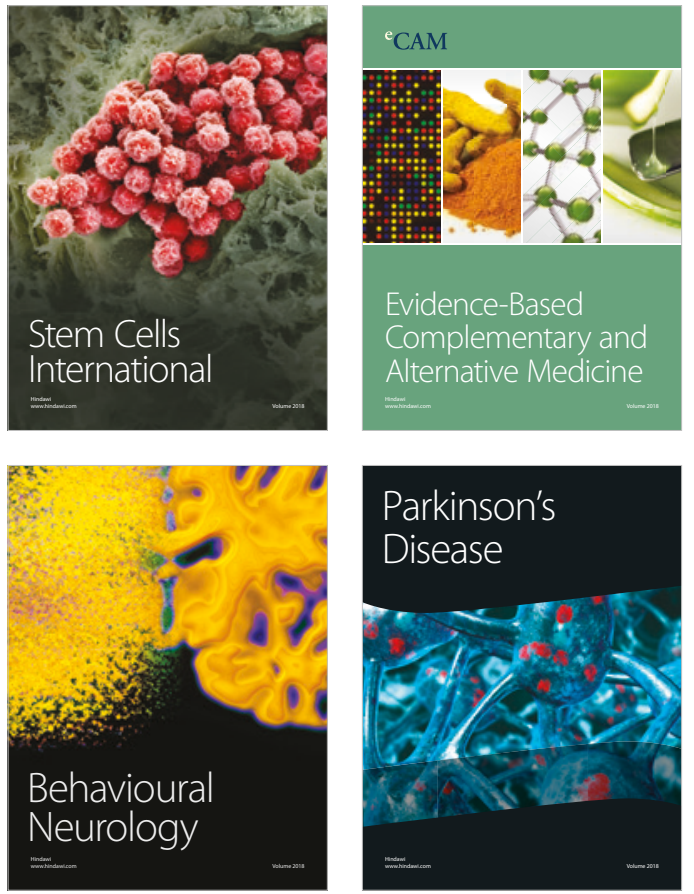

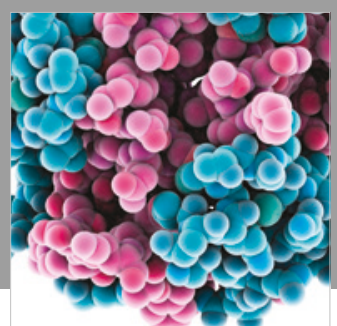

ournal of

Diabetes Research

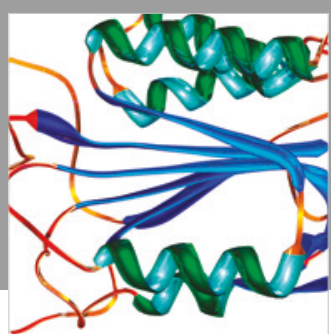

Disease Markers
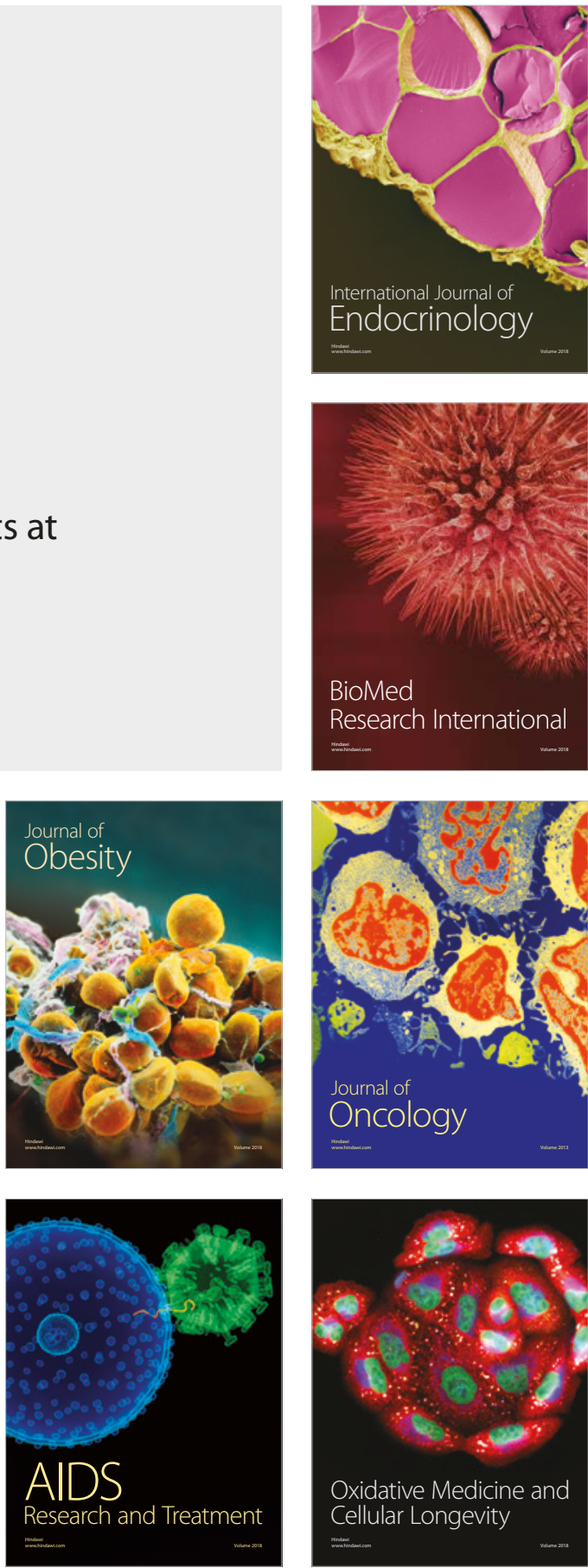